\title{
IDENTIFICACIÓN DE LOS CONECTORES DISCURSIVOS DE MÁS ALTA FRECUENCIA EN NOTAS PERIODÍSTICAS DEPORTIVAS ${ }^{1}$
}

\author{
IDENTIFICATION OF THE MOST FREQUENTLY USED \\ DISCOURSE CONNECTORS IN SPORTS NEWS STORIES
}

\author{
SARA QUINTERO RAMÍREZ \\ Universidad de Guadalajara \\ qsara@hotmail.com
}

\section{RESUMEN}

El presente estudio tiene como objetivo identificar los conectores discursivos más frecuentemente empleados en un corpus constituido por 300 notas deportivas de diferentes diarios de habla hispana, distribuidas equitativamente en cada uno de los siguientes rubros: béisbol, fútbol soccer, fútbol americano, baloncesto, tenis y deportes varios. Lo anterior con el propósito de determinar las relaciones lógicas que se producen con mayor asiduidad en el marco de los textos deportivos, más particularmente de las notas periodísticas. Esta investigación tiene la finalidad de contribuir a la caracterización lingüística del discurso deportivo en español. Los resultados del estudio demostraron que los conectores de finalidad fueron los más frecuentemente empleados en el corpus, pues de los 1.462 conectores discursivos registrados, 520 casos fueron de finalidad; mientras que los conectores de condición fueron los menos recurrentes con apenas 51 casos contabilizados.

Palabras clave: Conectores discursivos, cohesión textual, notas periodísticas deportivas, AntConc.

\section{ABSTRACT}

This study aims to identify the most frequently used discourse connectors in a corpus consisting of 300 different sports articles published in Spanish-language newspapers: 50 articles from baseball, football soccer, American football, basketball, and tennis, with a further 50 from other sports. The main purpose of the study consists of determining the

\footnotetext{
${ }^{1}$ Investigación vinculada al proyecto de "Estudio Lingüístico del Discurso Deportivo" del Cuerpo Académico: UDG-560: Traducción y lingüística aplicada a la enseñanza de lenguas, Universidad de Guadalajara.
} 
logical relationships that occur most frequently in the context of sporting texts, particularly in news stories. This research aims to contribute to the categorisation of linguistic sports discourse in Spanish. The results of the study showed that the connectors of purpose were the most frequently used in the corpus. Among the 1.462 discourse connectors found in the corpus, 520 tokens reflected purpose; whereas condition connectors were the least recurrent with only 51 tokens.

Keywords: Discourse connectors, textual cohesion, sports news stories, AntConc.

Recibido: 12.01.2015. Aceptado: 15.10.2015.

\section{INTRODUCCIÓN}

-1 deporte juega un rol primordial en los diferentes medios de comunicación, Cues a través de ellos logra atraer masas y ocasionar que los adeptos de un equipo o un atleta específico se propaguen considerablemente (Ubidia y Enrique, 2012: 31). De acuerdo con Medina Cano (2010: 164), los medios de comunicación han contribuido notablemente en la difusión del deporte, sobre todo en dos vertientes: la primera en cuanto a la formación de un imaginario respecto al deporte haciendo que éste se vuelva apasionante y la segunda en cuanto al establecimiento de un discurso muy particular que se distingue de otros por una serie de atributos esencialmente lingüísticos.

De acuerdo con Franco (2004: 98), las notas periodísticas deben tener una serie de cualidades, entre las cuales sobresalen la claridad, la exactitud y la precisión aunadas a la originalidad, la atracción y el color. Desde luego, las notas periodísticas deportivas tratan de respetar y seguir dichas características, en especial las tres últimas, ya que la diversidad de eventos deportivos produce constantemente noticias que pueden resultar novedosas, sensacionales e incluso memorables (Medina Cano, 2010: 164).

Respecto al deporte que se difunde masivamente a través de notas periodísticas, Medina Cano (2010: 174) afirma: "La prensa asume el papel del analista y del historiador. Es un documento para guardar para la posteridad con el registro escrito del encuentro que se inscribe en los anales y se transforma en historia. Los periodistas informan sobre lo acaecido, proclaman y valoran los momentos más sobresalientes del encuentro". Así pues, el periodista deportivo debe jugar un rol intermedio entre el rigor e inflexibilidad del discurso técnico y la creatividad e intuición del lenguaje subjetivo que hace hincapié en frases ceremoniosas y rodeos retóricos (Medina Cano, 2010: 197).

Con base en las elucidaciones de Franco (2004) y Medina Cano (2010), interesa saber cómo los periodistas logran enlazar las diferentes ideas técnicas y creativas en sus notas deportivas de forma clara y precisa, pero al mismo tiempo de manera 
original y atractiva. Así pues, el objetivo del presente estudio consiste en identificar los conectores discursivos de los que se sirven con mayor frecuencia los periodistas deportivos de habla hispana en un corpus de 300 notas periodísticas. De esta manera, se busca contribuir a la descripción lingüística del discurso deportivo en español.

A fin de alcanzar el objetivo planteado, es conveniente dar inicio a este artículo con un apartado teórico que permita elucidar el origen de los conectores discursivos, su relevancia a nivel del texto y algunas propuestas de clasificación por parte de los estudiosos del tema. Enseguida, se presenta la metodología explicando cómo se ha constituido el corpus, así como la manera en que se ha procedido para examinarlo. Por último, se exponen las conclusiones obtenidas mediante el presente estudio.

\section{FUNDAMENTOS TEÓRICOS}

\subsection{Cohesión textual}

De acuerdo con la lingüística textual, lo que hace a un texto ser texto no es su gramaticalidad, sino su textualidad. Desde la perspectiva de De Beaugrande y Dressler (1997: 11), un texto no es una mera suma de las palabras, oraciones o párrafos que lo componen; en realidad, un texto es considerado un acontecimiento comunicativo que sigue siete normas de textualidad (De Beaugrande y Dressler, 1997: 35). Las normas más estudiadas son aquéllas de orden lingüístico: cohesión y coherencia.

La cohesión, primera norma expuesta por De Beaugrande y Dressler (1997), evidencia las relaciones entre los diferentes elementos lingüísticos que componen un texto (Lozano, Peña-Marín y Abril, 1999). En palabras de Halliday y Hasan (1976), la cohesión es un concepto de orden semántico que hace referencia a las relaciones de significado que se establecen en el interior del texto y que precisamente definen al texto como tal. La cohesión se evidencia cuando la interpretación de un elemento discursivo depende justamente de la interpretación de otro elemento discursivo. Así pues, la cohesión se encarga de asegurar las conexiones al interior del texto a fin de expresar las relaciones semánticas entre los diferentes segmentos constitutivos textuales.

De acuerdo con Sánchez Avendaño (2005), existe una diversidad de mecanismos de cohesión que se encargan de proporcionarle al texto unidad. "Dentro de estos mecanismos de cohesión textual, los llamados 'conectores discursivos' son los recursos que más evidentemente actúan como señalizaciones o indicios superficiales de los lazos semánticos profundos existentes entre porciones del texto" (Sánchez Avendaño, 2005: 171). Así pues, el presente estudio se enfoca en la frecuen- 
cia de los conectores discursivos en el marco de textos periodísticos deportivos.

\subsection{Conectores discursivos}

"Los conectores son palabras de clase cerrada, generalmente conjunciones o adverbios, que funcionan como un 'pegamento semántico' entre diversas unidades lingüísticas del discurso" (De Vega, 2005: 86). Los conectores discursivos juegan un rol importante en la construcción del significado del discurso. De acuerdo con Martín Zorraquino y Portolés Lázaro (1999), los conectores tienen la tarea de relacionar semántica y pragmáticamente dos elementos del discurso, esto es secuencias de constituyentes que exceden el límite de unidades como la palabra, la frase o la oración. En efecto, De Vega (2005: 86) asevera que "los conectores no se limitan a indicar que existe una relación formal (v.g., sintáctica) entre dos unidades lingüísticas, sino que inducen al lector a construir un tipo de relación semántica particular entre dos eventos".

A fin de proponer una clasificación de los conectores, Domínguez García (1997: 35) señala que ésta no puede establecerse siguiendo criterios meramente gramaticales, pues un rasgo distintivo de los conectores es precisamente su aportación de valor conceptual a la relación entre enunciados. De tal suerte que un considerable número de autores como Martín Zorraquino y Portolés Lázaro (1999) clasifican los conectores en tres tipos esenciales, a saber: aditivos, consecutivos y contraargumentativos. Por su parte, Calsamiglia y Tusón (2002) además de considerar los conectores aludidos por Martín Zorraquino y Portolés Lázaro, agregan a la lista conectores de causa, condición, finalidad, así como de espacio y tiempo. Los autores del presente artículo coinciden con la clasificación concebida por Calsamiglia y Tusón (2002), sin embargo, para el trabajo en cuestión el foco estará únicamente en seis conectores: aditivos, consecutivos, contraargumentativos, causales, condicionales y finales ${ }^{2}$.

"Los conectores aditivos enlazan dos porciones informativas con la misma orientación discursiva" (Sánchez Avendaño, 2005: 175). De acuerdo con De Vega (2005: 86), estos conectores son polisémicos y presentan pocas restricciones semánticas, de tal suerte que dependiendo del cotexto y el contexto, pueden asegurar una diversidad de significados: enumeración, sucesión temporal, causa e, incluso, adversidad. Algunos conectores aditivos propuestos por Montolío (2001: 143) son: asimismo, igualmente, de igual manera, además, encima, incluso, inclusive, etc. Cabe señalar que la mayoría de los autores que hablan de conectores aditivos

\footnotetext{
${ }^{2}$ Hemos decidido dejar de lado los espaciales y temporales por cuestiones de espacio y porque nos gustaría dedicarles un estudio especial a estos dos, ya que se enfocan más en materia descriptivonarrativa.
} 
no presentan la conjunción y como conector (Martín Zorraquino y Portolés Lázaro, 1999; Montolío, 2001; Calsamiglia y Tusón, 2002; entre otros); de tal suerte que en el presente estudio tampoco se considera ${ }^{3}$. El ejemplo (1) presenta una relación aditiva entre dos segmentos textuales de una nota sobre béisbol que tienen una misma orientación discursiva a través del conector además.

(1) Fue hasta el sexto rollo que los Brewers pudieron conectar de hit cuando Elian Herrera sacó elevado al jardín central, el patrullero perdió la pelota en el sol y cayó para romper el maleficio, además el corredor aprovechó lo anterior para llegar hasta tercera. [Padres vence a Cerveceros con actuación redonda, La Afición, 07/03/2014].

"En la coordinación consecutiva se combinan dos oraciones mediante un signo conector que unifica en enunciado sus respectivas significaciones, ordenándolas en una relación deductiva o de 'causa $\rightarrow$ efecto'. De este modo, la segunda oración se convierte en un derivado casi natural de la primera, cualesquiera que fueran los significados de cada una" (Álvarez, 1999: 3789). "Los conectores consecutivos cumplen la función de indicar que la porción informativa en la que aparecen se debe procesar como una consecuencia de la porción anterior" (Sánchez Avendaño, 2005: 184).

Para Montolío (2001: 101), los conectores consecutivos evidencian la relación de causa-consecuencia, ofreciendo al receptor del texto una demostración o conclusión a través de una premisa o argumento concreto. De acuerdo con la autora, hay conectores consecutivos que están integrados a la oración y que por ende, contienen la conjunción que, tales como: de manera que, de modo que, asi que, por lo que, de ahi que, etc. Asimismo, existen conectores de tipo parentético tales como: por ello, por esa razón, por lo tanto, en consecuencia, por consiguiente, etc. En el ejemplo (2) el conector integrado por lo que sirve para manifestar la consecuencia de la primera cláusula Walcott se rompió el ligamento [...].

(2) El habilidoso elemento del Arsenal salió lesionado en el clásico de Londres ante el Tottenham Hotspur. Walcott se rompió el ligamento cruzado anterior de la rodilla izquierda el pasado 4 de enero, por lo que se baja del barco inglés para disputar el torneo mundialista. [Jugadores que corren peligro de no llegar a Brasil, Excélsior, 28/03/2014].

Los conectores contraargumentativos establecen una relación en la que lo presentado en el segundo segmento textual contrasta o limita lo expresado en el pri-

\footnotetext{
${ }^{3}$ Además, no es nuestro propósito examinar las 2.473 recurrencias de $y$ en nuestro corpus a fin de dar cuenta de cuántas ocasiones dicha conjunción actúa en labor de conector aditivo de cláusulas.
} 
mer segmento. En otras palabras, establecen una relación supresora o atenuadora de alguna conclusión deducible del primer segmento del texto (De Vega, 2005: 90). Algunos autores como Algeo (1973) hacen la distinción entre los conectores adversativos y los concesivos. Otros como Murray (1997) y Soria (2005) no hacen distinción entre ambos conectores y en su lugar, proponen el término de adversatividad que engloba a ambos.

Por su parte, Flamenco García (1999: 3809) asevera lo siguiente: "Las construcciones concesivas y adversativas hacen referencia a dominios nocionales muy próximos. Esta cercanía semántica o parentesco lógico, como señalaba la tradición gramatical, se pone de manifiesto en que es posible parafrasear una mediante la otra, y en particular a las más representativas: las encabezadas por las conjunciones aunque y pero respectivamente". Siguiendo a Murray (1997), Flamenco García (1999) y Soria (2005), se considera que los conectores concesivos y adversativos están en un mismo rubro, el de la contraargumentación. En el ejemplo (3) advertimos el conector aunque integrado al segundo segmento textual para evidenciar el contraargumento respecto al primer segmento en una nota de fútbol americano.

(3) Sharper fue acusado de violación, pagó 200.000 dólares aunque deberá presentarse ante un tribunal el 17 de febrero. [Arrestado Darren Sharper, ex astro de la NFL, La Afición, 18/01/2014].

Los conectores causales, como su nombre lo indica, ponen de relieve la causa en la relación de causa-efecto. Dichos conectores no son reconocidos como tales por Martín Zorraquino y Portolés Lázaro (1999) ni por Montolío (2001), pero sí son considerados conectores por Calsamiglia y Tusón (2002) así como por De Vega (2005). De hecho, Calsamiglia y Tusón (2002) conciben una categoría que comprende los conectores de base causal, esto es causales, consecutivos y finales. Se cree conveniente considerar estos tres tipos de conectores para el presente estudio, pues al igual que los tres anteriores, es decir aquéllos de adición, consecuencia y contraargumentación, éstos también se encargan de evidenciar relaciones lógicas.

De acuerdo con criterios sintácticos, semánticos y pragmáticos, Galán Rodríguez (1999) propone considerar dos grupos de oraciones causales: causales integradas y causales periféricas. Por un lado, las causales integradas tienen un mayor grado de dependencia respecto al verbo de la oración principal que las segundas. Asimismo, las causales integradas se encuentran precisamente integradas al predicado verbal, mientras que las causales periféricas no.

Las causales periféricas, por su parte, "presentan un hecho (B) como explicación más o menos razonable de otro hecho (A). Si la oración introduce una información 'temática' (conocida), ocupa la posición inicial de forma preferente (ya que, visto que, puesto que, supuesto que) u obligatoria (como). Si la información es 'remática' (nueva), la oración va pospuesta (que, porque, pues)" (Galán Rodríguez, 
1999: 3608). Sin embargo, esta configuración depende esencialmente de la intención comunicativa del interlocutor y del énfasis que éste quiera dar a las diferentes partes de su mensaje. En el ejemplo (4) porque es el conector que antecede justamente la cláusula causativa los locales presionaron mucho [...] y es precedido por la cláusula de consecuencia No fue fácil la victoria.

(4) El Barça también ganó, en este caso en el campo del Espanyol, y sufriendo mucho. No fue fácil la victoria, porque los locales presionaron mucho en todo momento y contaron con la permisividad de Clos Gómez para pasar en más de un momento la fina línea que separa el juego intenso del juego duro. [Victorias del Barça, Atlético y Real Madrid, Sport, 29/03/2014].

Los conectores de condición introducen una idea de necesidad o restricción estableciendo una relación entre dos segmentos, uno de ellos resulta requisito para que el otro pueda cumplirse. "La condición lógica es la relación en la que el hablante propone una situación en la que si la primera de las proposiciones (la prótasis) es real, la segunda proposición (la apódosis) será real también. Si $p$, q" (Quintero Ramírez, 2013: 5).

De acuerdo con Montolío (1999: 3647), las construcciones condicionales engloban un numeroso grupo de diversas estructuras sintácticas. La construcción más prototípica es aquélla introducida por el conector $s i$, aunque evidentemente no la única. Asimismo, la autora advierte que la noción de condición puede estar relacionada con otras nociones tales como tiempo, causa, finalidad, comparación, contraste, concesión, etc. Algunos ejemplos de estos conectores son: si, en caso de, con tal de que, siempre que, a menos que, entre otros. En el ejemplo (5) observamos el prototípico conector si en el marco de una cita directa enunciada por el beisbolista Mike Trout; el conector se ubica en la prótasis para indicar la condición.

(5) Mike Trout no quiso hablar sobre la posibilidad de un contrato multianual: "Simplemente salgo a jugar", señaló. "Si el dinero está donde ocurren las cosas importantes, entonces ahí es donde va a estar". [Los Angelinos chiquean a su estrella, El Informador, 27/02/2014].

Los conectores de finalidad no son considerados ni por Martín Zorraquino y Portolés Lázaro (1999) ni por Montolío (2001), pero sí por Calsamiglia y Tusón (2002) que los definen como aquellos que evidencian la meta o el propósito que se pretende alcanzar (Calsamiglia y Tusón 2002: 248). Galán Rodríguez (1999: 3621) señala que las oraciones finales al evidenciar el propósito se interpretan como un evento posterior a otro; es por ello que comúnmente se ubican en la segunda posición de la secuencia, salvo cuando se quiere hacer hincapié en el objetivo designado. Algunos ejemplos de conectores propuestos por esta autora son: 
para (que), a fin de (que), con el \{propósito/motivolobjeto\} de (que), con el fin de (que), entre otros. En el ejemplo (6) se observa el prototípico conector para seguido de un sintagma de infinitivo que evidencia finalidad en una nota de básquetbol.

(6) Los turcos supieron leer con inteligencia el encuentro y obligaron a los de Krzyzewski a bajar a la tierra para llevarse la victoria. [Turquía 'humaniza' al Dream Team y acaba aplastada por las torres Faried-Davis, Marca, 31/08/2014].

\section{METODOLOGÍA}

\subsection{Constitución del corpus}

En este estudio se trabajó en base a la siguiente pregunta de investigación: ¡cuáles son los conectores discursivos que se emplean más asiduamente en un corpus de 300 notas deportivas de diferentes diarios de habla hispana? A través de la respuesta a dicha pregunta se busca determinar las relaciones lógicas que se producen con mayor reiteración en el marco de los textos deportivos, más particularmente de las notas periodísticas. Asimismo, con dicha respuesta se pretende contribuir a la descripción lingüística del discurso deportivo en español, más concretamente en el ámbito de las estrategias utilizadas por los periodistas deportivos para la junción de cláusulas.

Tal como se ha mencionado, para el presente estudio se cuenta con un corpus de 300 notas periodísticas deportivas, tomando 50 notas de cada uno de los siguientes rubros: béisbol, fútbol soccer, fútbol americano, baloncesto, tenis y deportes varios. Se consideró este último rubro de 'deportes varios' para agrupar notas de deportes cuyos eventos no son tan frecuentemente publicados en los diarios como aquéllos de los cinco deportes anteriores. Así pues, en el grupo de 'deportes varios' se incluyen eventos de automovilismo, boxeo, ciclismo, clavados, esquí, golf, natación, patinaje artístico, taekwondo, tiro con arco y toros.

Las notas periodísticas del corpus se obtuvieron de la sección deportiva de una diversidad de diarios de diferentes países de habla hispana en su versión electrónica, a saber: Excélsior, La Jornada, El Informador, Récord, Estadio, Esto, La Afición y El Universal de México; El Día, Terra Argentina, La Capital y Hoy de Argentina; La Vanguardia, Sport, As, La Razón y Mundo deportivo de España; El País y La Nación de Uruguay; El Nuevo Día de Puerto Rico; Deporte 10 de Paraguay; El Tiempo de Colombia y por último, Peru21 de Perú. Todas las notas deportivas fueron publicadas en sus respectivos diarios durante los meses de enero a diciembre de 2014 . Por último, cabe mencionar que el corpus completo está constituido de 99,600 palabras en total. 


\subsection{Procedimiento de análisis del corpus}

A fin de determinar la presencia y frecuencia de los conectores discursivos en las notas deportivas del corpus aludido en el apartado anterior, se utilizó el programa de análisis de corpus $A n t C o n c^{4}$ diseñado por Laurence Anthony. Las herramientas de AntConc que se emplearon para el presente estudio fueron aquéllas de Concordancer y View Files.

La herramienta de concordancia permitió encontrar todos los casos de los conectores discursivos que habían sido empleados en los artículos deportivos del corpus, desplegando su cotexto inmediato. Posteriormente, a fin de tener una noción más vasta del cotexto en el que se empleaban los conectores y de poder definir su valor discursivo de manera más precisa, se recurrió a la herramienta de vista de archivos que muestra el conector empleado en el texto original. Cabe señalar que esta última herramienta fue utilizada de manera recurrente a fin de examinar el funcionamiento específico de un considerable número de conectores.

De manera simultánea al uso del programa AntConc, se buscó clasificar los conectores en base a los criterios expuestos por los estudiosos del tema en el apartado de fundamentos teóricos, en particular por Martín Zorraquino y Portolés Lázaro (1999) y por Calsamiglia y Tusón (2002), complementando con otros especialistas como Galán Rodríguez (1999), Montolío (2001) y Sánchez Avendaño (2005). Así pues, la clasificación de conectores considerada para el estudio fue la siguiente: a) aditivos, b) consecutivos, c) contraargumentativos, d) causales, e) condicionales y f) finales. Por último, se prestó atención a las frecuencias tomando en cuenta la clasificación aludida con el objeto de determinar el tipo de conector más productivo en el corpus.

\section{ANÁLISIS}

Luego de exponer la metodología del presente estudio, es preciso dar paso al análisis del corpus. A través del programa AntConc se pudo localizar 1.462 conectores discursivos, de los cuales 91 fueron de adición, 63 de consecuencia, 499 de contraargumentación, 238 de causa, 51 de condición y 520 de finalidad. A continuación se presentan de manera detallada nuestros hallazgos respecto a cada uno de los tipos de conectores de esta investigación.

\footnotetext{
4 "AntConc is a freeware, multi-platform, multi-purpose corpus analysis toolkit [...] It hosts a comprehensive set of tools including a powerful concordancer, word and keyword frequency generators, tools for cluster and lexical bundle analysis, and a word distribution plot" (Anthony, 2004: 7).
} 


\subsection{Conectores de adición}

En el marco de los conectores de adición se consideraron las propuestas de Martín Zorraquino y Portolés (1999) y Montolío (2001). Por un lado, los primeros proponen los siguientes conectores: además, encima, aparte, por añadidura, incluso, inclusive y es más. Por su parte, la segunda autora propone dos tipos de conectores: a) aquellos que introducen algo nuevo pero sin valorarlo desde una perspectiva argumentativa $\mathrm{y} \mathrm{b}$ ) aquellos que introducen algo nuevo valorándolo como más fuerte que lo anterior. En el primer rubro, Montolío (2001) agrupa: asimismo, igualmente, de igual maneralmodolforma, del mismo modo y de la misma maneral forma. En el segundo grupo, incluye: además, encima, es más, por añadidura, incluso e inclusive, es decir todos los conectores propuestos por Martín Zorraquino y Portolés Lázaro (1999), salvo aparte. Para el presente trabajo se han considerado los conectores propuestos por los tres autores.

Así pues, en el corpus se encuentran 91 conectores de adición. El más recurrente resultó además con 69 frecuencias. Enseguida, se ubica el conector incluso con 14 frecuencias. Otros conectores presentes en el corpus fueron: asimismo con 4 recurrencias, igualmente con 2, de igual forma con 1 y es más también con 1 . Conectores como del mismo modo, de la misma maneralforma, encima y por añadidura no aparecieron en el corpus. En la Tabla I se aprecia más claramente la distribución de las frecuencias de los conectores aditivos.

Tabla I. Conectores de adición.

\begin{tabular}{|l|l|}
\hline Conectores & Frecuencias \\
\hline Además & $69(75.82 \%)$ \\
\hline Incluso/ inclusive & $14(15.38 \%)$ \\
\hline Asimismo & $4(4.4 \%)$ \\
\hline Igualmente & $2(2.2 \%)$ \\
\hline De igual manera/modo/forma & $1(1.1 \%)$ \\
\hline Es más & $1(1.1 \%)$ \\
\hline Del mismo modo, de la misma manera/forma & - \\
\hline Encima & - \\
\hline Por añadidura & - \\
\hline Total & $91(100 \%)$ \\
\hline
\end{tabular}

El ejemplo (7) está constituido por un fragmento de una nota de baloncesto en donde se observa la presencia de dos conectores de adición, asimismo y además, 
que sirven para dar continuidad a las declaraciones de la deportista Laia Palau. El primer conector sirve para darle cohesión al texto, pues enlaza la cita anterior y la paráfrasis posterior a él. El segundo vuelve a dar cohesión al texto y se emplea para introducir nuevamente una cita de la basquetbolista en la que relata una anécdota. En el ejemplo (8) se advierte la presencia del conector incluso para enlazar dos actos de habla que realiza la protagonista de la nota periodística Effy Sánchez: detalló... y agregó...

(7) Laia Palau también advirtió lo siguiente: "Tenemos que mirar a esta final y aprender porque no queremos quedarnos aquí". Asimismo, recordó que participar en los Juegos Olímpicos de Río de Janeiro pasa por hacer "un buen europeo el año que viene". Además, Palau comentó una de las anécdotas de la final: "La entrenadora asistente hizo un vídeo asemejándonos a cada una de nosotras con los dibujos de la Warner Bros y lo clavó". [Alba Torrens: "La plata demuestra el gran trabajo del baloncesto femenino", 20 minutos, 6/10/2014].

(8) Effy Sánchez, presidenta de la Federación Mexicana de Tiro con Arco, detalló que serán 22 los estados representados, incluso, agregó, se darán cita exponentes de Sinaloa y Veracruz, que empiezan a crear escuela en dicho deporte. [Los arqueros disputan lugares en el Nacional de Exteriores, Esto, 11/03/14].

\subsection{Conectores consecutivos}

Como se mencionó en el apartado de los fundamentos teóricos, de acuerdo con Montolío (2001), estos conectores pueden escindirse en dos grandes apartados: a) aquellos que están integrados a la oración y b) los parentéticos. En el primer apartado la autora agrupa: asi que, de maneralmodo que, por lo que y de ahi que. En el segundo grupo incluye: por ello/eso, por eseltalldicho motivo/razón/causa, por (lo) tanto, en consecuencia, por consiguiente, por ende, pues y asi pues. Calsamiglia y Tusón (2002) añaden al listado propuesto por Montolío (2001) los siguientes conectores: luego, de ello resulta que, asi que, de donde se sigue, de suerte que, en efecto y entonces. Para el estudio se han considerado todos los conectores propuestos por las tres autoras.

En el corpus se registraron 63 conectores consecutivos, de los cuales por lo que fue el más recurrente con 29 frecuencias, por elloleso con 16, asi que con 11, entonces empleado con valor de consecuencia con 4 , de manera que con 2 y de ahi que con 1 frecuencia. Los conectores de ello resulta (que), por eseltalldicho motivo/razón/ causa, por (lo) tanto, en consecuencia, por consiguiente, por ende, de donde se sigue, de suerte que, en efecto y asi pues no se encontraron en el corpus. Por último, si bien los 
conectores pues y luego se emplean con gran reiteración en el corpus, no figuraron con valores de consecuencia. La Tabla II sintetiza los resultados encontrados en relación a los conectores consecutivos.

Tabla II. Conectores de consecuencia.

\begin{tabular}{|l|l|}
\hline Conectores & Frecuencia \\
\hline Por lo que & $29(46.03 \%)$ \\
\hline Por ello/ eso & $16(25.4 \%)$ \\
\hline Así que & $11(17.46 \%)$ \\
\hline Entonces & $4(6.35 \%)$ \\
\hline De manera/ modo que & $2(3.17 \%)$ \\
\hline De ahí que & $1(1.59 \%)$ \\
\hline De ello resulta (que) & - \\
\hline Por ese/ tal/ dicho motivo/razón/causa & - \\
\hline Por (lo) tanto & - \\
\hline En consecuencia & - \\
\hline Por consiguiente & - \\
\hline Por ende & - \\
\hline De donde se sigue & - \\
\hline De suerte que & - \\
\hline En efecto & - \\
\hline Así pues & - \\
\hline Pues & - \\
\hline Luego & $-100 \%)$ \\
\hline Total & - \\
\hline
\end{tabular}

En el ejemplo (9) se observa el empleo del conector integrado por lo que, que como bien señala Montolío (2001: 101) contiene la conjunción que a fin de enlazar las dos oraciones que conforman el párrafo y corroborar una idea de consecuencia entre ellas. En (10) se aprecia el uso del conector parentético por eso que evidencia la consecuencia respecto a la oración anterior sin integrarse. Por último, en (11) se advierte la presencia del conector integrado asi que, que pone de manifiesto la relación de causa-consecuencia entre la oración precedente y la ulterior. 
(9) El pelotero dominicano sufre de una lesión en el tendón de la corva, por lo que estará inactivo durante quince días. [Furcal, inhabilitado por los Marlins, El Universal, 30/03/14].

(10) La situación económica de la Fiesta es delicada, por eso todos debemos buscar fórmulas para conseguir que los festejos sean viables, partiendo de las corridas pero especialmente con las novilladas. [Las 17 alternativas que nos deja 2014, La Razón, 28/12/14].

(11) Las negociaciones para reestructurar su contrato fallaron, asi que la liberación le ahorra a Dallas 7.4 millones y da a la franquicia nueve millones para gastar en la agencia libre. [Dallas le da las gracias a DeMarcus Ware, $E l$ Informador, 12 /03/14].

\subsection{Conectores contraargumentativos}

En el marco de la contraargumentación, Calsamiglia y Tusón (2002) proponen cuatro subtipos de conectores: a) oposición, b) sustitución, c) restricción y d) concesión. Entre los primeros plantean: pero, en cambio, sin embargo y ahora bien. Como oposición, proponen: sino, en lugarlvez de, por el contrario, antes bien y contrariamente. En el tercer grupo incluyen: excepto si y a no ser que. Finalmente, en el último grupo presentan: de todos modos, sea como sea, en cualquier caso, a pesar de, no obstante, con todo, aun asi, después de todo y asi y todo.

Por su parte, Montolío (2001: 49) concibe también cuatro grupos de conectores contraargumentativos. Desde una perspectiva sintáctica, propone dos grupos: conectores integrados y conectores parentéticos. Los primeros se unen a la cláusula mediante la conjunción que o las preposiciones a y de como: aunque, a pesar de (que), pese a (que) y si bien 5 . Los parentéticos se insertan entre las cláusulas y tienen más oportunidad de desplazamiento, tal es el caso de pero, sin embargo, no obstante, ahora bien, con todo, aun así y a pesar de todo.

Desde una perspectiva semántica, la autora propone un tercer grupo que, independientemente de sus rasgos integradores o parentéticos, el segundo segmento despliega un significado de corrección respecto a algo expuesto en el primer segmento; ejemplos de éstos serían: mientras que, en tanto que, sino que, en cambio, por el contrario y antes bien. El último grupo está conformado por conectores que no son realmente contraargumentativos, pero que en diversos contextos despliegan un valor semejante, por ejemplo: de todas formas, de todas maneras y de todos modos. Para el estudio del corpus se han considerado todos los conectores aquí expuestos.

En el corpus se registraron 499 conectores contraargumentativos, de los cuales pero fue el más frecuente con 299 recurrencias. Otros conectores que aparecieron

\footnotetext{
${ }^{5}$ Aunque éste no se encuentre seguido de conjunción o preposición.
} 
con cierta asiduidad fueron mientras que con 52 frecuencias, sin embargo con 41, a pesar de con 39, pese a con 22, sino con 17. Cabe señalar que de las 39 ocasiones que se observó a pesar de en el corpus, 13 fueron seguidas de la conjunción que. Asimismo, de las 22 ocasiones que se identificó pese a, 9 fueron seguidas de la misma conjunción. Igualmente, de las 17 veces que se registró sino, 8 de ellas fueron seguidas de que. Menos repetidamente se identificó no obstante con 6 recurrencias, en lugarlvez de y con todo con 5 cada uno, aun asi y si bien con 4 recurrencias cada uno, en tanto que con 2 y después de todo, por el contrario y sea como sea con sólo 1 cada uno. En cambio, ahora bien, antes bien, contrariamente, excepto si, a no ser que, de todos modos, de todas maneras/formas, en cualquier caso y asi y todo no figuraron en el corpus. La Tabla III resume los datos antes aludidos.

Tabla III. Conectores de contraargumentación

\begin{tabular}{|c|c|}
\hline Conector & Frecuencia \\
\hline Pero & $299(59.92 \%)$ \\
\hline Mientras que & $52(10.42 \%)$ \\
\hline Sin embargo & $41(8.22 \%)$ \\
\hline A pesar de & $\begin{array}{l}39(7.82 \%) \\
{[13 \text { que }(2.6 \%)}\end{array}$ \\
\hline Pese a & $\begin{array}{l}22(4.41 \%) \\
{[9 \text { que] }(1.8 \%)}\end{array}$ \\
\hline Sino & $\begin{array}{l}17(3.41 \%) \\
{[8 \text { que] }(1.6 \%)}\end{array}$ \\
\hline No obstante & $6(1.2 \%)$ \\
\hline En lugar/vez de & $5(1 \%)$ \\
\hline Con todo & $5(1 \%)$ \\
\hline Aun así & $4(0.8 \%)$ \\
\hline Si bien & $4(0.8 \%)$ \\
\hline En tanto que & $2(0.4 \%)$ \\
\hline Después de todo & $1(0.2 \%)$ \\
\hline Por el contrario & $1(0.2 \%)$ \\
\hline Sea como sea & $1(0.2 \%)$ \\
\hline En cambio & - \\
\hline Ahora bien & - \\
\hline Antes bien & - \\
\hline Contrariamente & - \\
\hline Excepto si & - \\
\hline
\end{tabular}




\begin{tabular}{|l|c|}
\hline A no ser que & - \\
\hline De todos modos & - \\
\hline De todas maneras/ formas & - \\
\hline En cualquier caso & - \\
\hline Así y todo & - \\
\hline Total & $499(100 \%)$ \\
\hline
\end{tabular}

En (12) se encontró el conector pero que resultó por mucho el más frecuente de esta sección. En el ejemplo, este conector calificado como conector de oposición por Calsamiglia y Tusón (2002) y como conector parentético por Montolío (2001: 49) se emplea para contrastar la situación de los ciclistas que habían logrado separarse del grupo y la sorpresa de encontrar el camino cerrado.

(12) Casi llegando a Tepoztlán varios fueron los corredores que comenzaban a separarse del grupo, pero tuvieron la sorpresa de que el camino trazado estaba cerrado ya que fue Miércoles de Ceniza y los feligreses que acudieron a la iglesia ocupaban el espacio donde pasaría el contingente, por lo que cambiaron a otro carril más pedregoso, que les quitó segundos valiosos en la competencia. [Prado mostró su grandeza en la segunda fase de la Vuelta Ciclista, Esto, 06/03/14].

En el ejemplo (13) se observa un fragmento de una nota tenística en la que se emplean dos conectores contraargumentativos por el contrario y a pesar de. El primero con rasgos parentéticos y de oposición, tal como lo señala Montolío (2001: 49), sirve para establecer una suerte de parangón y a la vez de corrección entre el segmento anterior que expone la situación de Rafael Nadal en el partido y el segmento posterior que presenta la situación de Novak Djokovic. El segundo conector de concesión, a pesar de, está integrado al segmento que le sigue y se emplea para enlazar las dos cláusulas subsiguientes, esto es el riesgo que corrió en su golpeo y el serbio ganó el 83 por ciento [...]

(13) En declaraciones previas a la final, Nadal dijo que su servicio iba a ser clave y tenía que jugar por encima de su nivel para poder ganar al serbio, y hoy no tuvo su día con el saque y apenas dio una fracción de su habitual imagen. Por el contrario, y a pesar del riesgo que corrió en su golpeo, el serbio ganó el 83 por ciento de sus puntos en sus primeros servicios y cometió dos errores no forzados menos que el español. [Djokovic vence a Nadal y logra su cuarto título en Miami, Sport, 03/03/14]. 


\subsection{Conectores causales}

De acuerdo con Sánchez Avendaño (2005: 187), existe una fuerte asociación entre las relaciones lógicas causales y consecutivas, de tal suerte que resulta difícil proponer clasificaciones categóricas respecto al valor de los conectores. Sin embargo, Galán Rodríguez (1999) propone los siguientes conectores: porque, ya que, visto que, puesto que, supuesto que, como y pues. Por su parte, Calsamiglia y Tusón (2002) añaden los siguientes: a causa de ello, por eso, dado que, por el hecho de que, en virtud de y gracias a. Para el presente estudio se han considerado todos los conectores propuestos por las tres autoras.

En el corpus se contabilizaron 238 conectores de causa, de los cuales porque fue el más repetidamente empleado con 88 frecuencias. Otros conectores que aparecieron con cierta reiteración en el corpus fueron ya que con 48 recurrencias, pues con 46, gracias a con 30 y por eso con 11 . Menos asiduamente se ubicó a causa de con 7 frecuencias, puesto que con 6 y dado que y por el hecho de que con 1 frecuencia cada uno. Respecto a este último conector, cabe señalar que se encontró empleado sin la preposición de, esto es por el hecho que. Los conectores visto que, supuesto que, como y en virtud de no figuraron en el corpus. La Tabla IV esquematiza las frecuencias de los conectores causativos.

Tabla IV. Conectores de causa.

\begin{tabular}{|l|c|}
\hline Conector & Frecuencia \\
\hline Porque & $88(36.97 \%)$ \\
\hline Ya que & $48(20.17 \%)$ \\
\hline Pues & $46(19.33 \%)$ \\
\hline Gracias a & $30(12.61 \%)$ \\
\hline Por eso & $11(4.62 \%)$ \\
\hline A causa de (ello) & $7(2.94 \%)$ \\
\hline Puesto que & $6(2.52 \%)$ \\
\hline Dado que & $1(0.42 \%)$ \\
\hline Por el hecho (de) que & $1(0.42 \%)$ \\
\hline Visto que & - \\
\hline Supuesto que & - \\
\hline Como & - \\
\hline En virtud de & - \\
\hline Total & $238(100 \%)$ \\
\hline
\end{tabular}


En (14-16) se observa el uso de los conectores porque, ya que y pues, respectivamente, todos precedidos de un segmento textual que evidencia la consecuencia: el orgullo mostrado por el basquetbolista Ayón en (14), pelear en Nueva York como talismán en (15) y el agotamiento en los basquetbolistas colombianos en (16). Asimismo, los conectores de los ejemplos fueron seguidos de un segmento que da cuenta de la causa: la presencia de dos mexicanos en el Real Madrid en (14), el haber peleado 10 veces en Nueva York en (15) y la situación de que cuatro de los titulares de Colombia jugaron más de 35 minutos así como la profundidad de los brasileños en (16).

(14) Finalmente, se mostró orgulloso porque hay dos mexicanos en el Real Madrid, él en básquetbol y Javier "Chicharito" Hernández en el fútbol, curiosamente ambos con el número 14 en su espalda. [Ayón duda en regresar a selección mexicana de básquetbol, El Universal, 07/03/14].

(15) Cotto expresó que pelear en Nueva York le da suerte, ya que ha sostenido 10 peleas, ocho de ellas en el Garden. ["Maravilla" y Cotto, ansiosos, Esto, 03/06/14].

(16) El agotamiento se notó en los basquetbolistas del equipo colombiano, pues cuatro de sus titulares, estuvieron por más de 35 minutos en cancha y la profundidad de los brasileños pesó lo suficiente como para sacar 20 puntos de diferencia, 70-90. [Segunda derrota de Guerreros en la Copa Suramericana de baloncesto, El Tiempo, 22/10/14].

\subsection{Conectores condicionales}

De acuerdo con Montolío (1999: 3647), "las estructuras condicionales son una de las principales vías lingüísticas de las que dispone el individuo para expresar su capacidad de imaginar situaciones diferentes a las reales; de crear mundos posibles; de soñar con situaciones pasadas que podrían haber sido diferentes; de ocultar lo factual tras la apariencia de lo contingente". La citada autora estudia las diferentes posibilidades sintácticas que tiene el prototípico conector condicional si, al igual que algunos otros conectores, en particular a nivel de los tiempos y modos verbales, pues a través de éstos se hace hincapié en la posibilidad y expectativa de cumplimiento denotadas por el usuario de la lengua. Para el presente estudio se han considerado los conectores que Calsamiglia y Tusón (2002) proponen como condicionales y que se listan a continuación: si, con tal que, cuando, en el caso de que, según, a menos que, siempre que, mientras, a no ser que, siempre y cuando, sólo que y con que.

En el corpus se han encontrado 51 conectores de condición, de los cuales si ha sido el más recurrente con 42 frecuencias; le siguen cuando con 7 repeticiones y 
siempre y cuando así como siempre que con 1 frecuencia cada uno. Los conectores condicionales mientras, según, a no ser que, sólo que, con tal que, a menos que, en el caso de que y con que no se registraron en el corpus.

Se considera que los conectores condicionales han sido los menos recurrentes del corpus porque, como bien señala Montolío (1999: 3647), se emplean para expresar situaciones diferentes a las reales y crear mundos posibles. Las notas periodísticas no tienen este cometido, sino por el contrario, tienen la función principal de dar cuenta de hechos reales; de tal suerte que dichos conectores no han sido tan frecuentemente empleados por los periodistas deportivos como el resto de los que se han examinado aquí. La Tabla $\mathrm{V}$ esquematiza los hallazgos.

Tabla V. Conectores de condición.

\begin{tabular}{|l|l|}
\hline Conector & Frecuencia \\
\hline $\mathrm{Si}$ & $42(82.35 \%)$ \\
\hline Cuando & $7(13.73 \%)$ \\
\hline Siempre y cuando & $1(1.96 \%)$ \\
\hline Siempre que & $1(1.96 \%)$ \\
\hline Mientras & - \\
\hline Según & - \\
\hline A no ser que & - \\
\hline Sólo que & - \\
\hline Con tal que & - \\
\hline A menos que & - \\
\hline En el caso de que & - \\
\hline Con que & - \\
\hline Total & $51(100 \%)$ \\
\hline
\end{tabular}

Los fragmentos textuales (17-19) ejemplifican las pocas muestras que se hallaron de relaciones lógicas de condición. Los conectores si, cuando y siempre y cuando respectivamente evidencian el supuesto o condición. En (17) y (19) los conectores de condición se ubican al inicio del segundo segmento textual; mientras que en (18) el conector aparece al inicio del primer segmento. Tal como se señaló anteriormente, Montolío (1999) hace hincapié en los tiempos y modos verbales que se despliegan a través del uso de estos conectores. Así pues, en los ejemplos aquí aludidos se observó que si es precedido de un futuro de indicativo: será..., seguido de un presente de indicativo: gana..., es decir: futuro de indicativo $+s i+$ presente de indicativo. Por su parte, cuando es seguido de dos segmentos en presen- 
te de indicativo: ves... y ves...; a manera de fórmula se concibe: cuando + presente de indicativo + presente de indicativo. Finalmente, siempre y cuando es precedido de futuro de indicativo: se celebrará... y es seguido de presente de subjuntivo: lo permita; a manera de fórmula resulta: futuro de indicativo + siempre y cuando + presente de subjuntivo. Se considera pues que las condiciones aquí formuladas despliegan una alta expectativa de cumplimiento en el mundo real por parte de los periodistas.

(17) El Madrid será líder en solitario si gana hoy en Kaunas, ya que el Efes (el primero en derrotar a los blancos esta temporada) perdió ayer en Kazán. [Lituania: gran cantera del baloncesto en Europa, $A s, 21 / 11 / 14$ ].

(18) "No nos sorprende lo que hace Peyton", dijo Julius Thomas. "Cuando ves cómo trabaja todos los días, ves lo que él mismo se exige. Afortunadamente, eso es para beneficio de nosotros". ["Manning rompe el récord de Brady en la NFL con 51 pases para touchdowns", La Jornada, 22/12/14].

(19) Protección Civil informó en el Complejo Leyes de Reforma, donde se efectúan los duelos de natación en esta justa mesoamericana, que por seguridad se canceló la competencia programada para las 13:30 horas de este lunes, misma que se celebrará mañana en el mismo horario, siempre y cuando el tiempo lo permita. [Suspenden actividad de nado sincronizado y natación por mal tiempo, Excélsior, 17/11/14].

\subsection{Conectores finales}

Galán Rodríguez (1999: 3621) afirma que "las oraciones finales manifiestan una relación de causa-efecto en la que el efecto se interpreta como posterior (propósito virtual), frente a la anterioridad (motivo realizado) que designan las causales. Por esta razón, las finales (al margen del nexo utilizado) ocupan preferentemente la segunda posición de la secuencia [...]”. Calsamiglia y Tusón (2002) proponen la siguiente lista de conectores finales: para (que), a fin de (que), con el propósitolobjeto de (que) y de tal modo (que). Galán Rodríguez (1999) añade a la lista: por seguido de sintagma de infinitivo, así como las fórmulas $a+$ sustantivo + de + infinitivo (o de que + subjuntivo) así como con + sustantivo + dela + infinitivo (o de que + subjuntivo), tales como a efectos de y con miras a, respectivamente. Para el estudio se consideraron los conectores propuestos por Calsamiglia y Tusón (2002) así como aquéllos de Galán Rodríguez (1999).

En el corpus se ubicaron 520 conectores de finalidad, de los cuales para (que) resultó el más frecuentemente empleado con 512 recurrencias, de las cuales 39 desplegaron la conjunción que + presente de subjuntivo, mientras que en el resto de las recurrencias se observó el conector seguido de sintagma de infinitivo. Los 
otros conectores que aparecieron en el corpus con valor de finalidad fueron: por con 6 recurrencias y con el fin de y con miras a con 1 recurrencia cada uno. Estos tres seguidos siempre de sintagma de infinitivo. Los conectores a fin de, con el propósito de, con el objeto de, con ánimo de, con vistas a, con idea de, con intención de, a efectos de, con motivo de y con pretexto de no figuraron en el corpus. La Tabla VI esquematiza de mejor manera lo encontrado.

Tabla VI. Conectores de finalidad.

\begin{tabular}{|l|l|}
\hline Conector & Frecuencia \\
\hline Para (que) & $\begin{array}{l}512(98.46 \%) \\
{[39 \text { que }](7.5 \%)}\end{array}$ \\
\hline Por & $6(1.16 \%)$ \\
\hline Con el fin de (que) & $1(0.19 \%)$ \\
\hline Con miras a & $1(0.19 \%)$ \\
\hline A fin de (que) & - \\
\hline Con el propósito de (que) & - \\
\hline Con el objeto de (que) & - \\
\hline Con ánimo de (que) & - \\
\hline Con vistas a & - \\
\hline Con idea de (que) & - \\
\hline Con intención de (que) & - \\
\hline A efectos de (que) & - \\
\hline Con motivo de (que) & - \\
\hline Con pretexto de (que) & - \\
\hline Total & $520(100 \%)$ \\
\hline
\end{tabular}

Los ejemplos (20-22) muestran fragmentos textuales en los que se emplean los conectores de finalidad para, por y con el fin de. En los tres fragmentos se observa, tal como señala Galán Rodríguez (1999: 3621), que los conectores se ubican en el segundo segmento de la secuencia, ya que el propósito o meta se interpreta como algo posterior. Asimismo, se advirtió que los tres conectores son seguidos de sintagmas de infinitivo: alcanzar 12 goles en total, hacerles soltar la pelota y definir en julio a la mejor selección [...], respectivamente. Lo anterior resultó lo más recurrente en el corpus, pues de las 520 construcciones finales, 481 presentaron sintagma de infinitivo; mientras que sólo se registraron 39 con la conjunción que seguida de una cláusula en presente de subjuntivo. 
(20) Alcanza Cristiano Ronaldo 28 goles en Liga, tras marcar el primero de los cinco tantos del Real Madrid al Rayo Vallecano, en un encuentro en el que el galés Gareth Bale firmó un doblete para alcanzar 12 goles en total, y Dani Carvajal su primer tanto oficial con el primer equipo. [Cristiano Ronaldo responde ante la presión de Diego Costa y Messi, Mundo deportivo, 30/03/14].

(21) La medida busca evitar "que los muchachos hagan lo posible por hacerle soltar la pelota (al cátcher) cuando tiene el plato", expresó el manager de los Rangers de Texas, Ron Washington. ["Nueva regla prohíbe al corredor chocar al cátcher", Récord, 24/02/2014].

(22) Así lo anunció el profesor Jesús David Quintero Azuara, vicepresidente de natación de la FMN, quien destacó que "tenemos un grupo de 26 mujeres y 23 hombres con los que estamos trabajando actualmente, con el fin de definir en julio a la mejor selección que peleará los centroamericanos de Veracruz en noviembre", indicó. [Nadadores al Circuito Mexicano, Esto, 10/03/14].

\section{CONCLUSIONES}

Luego de observar cada uno de los subapartados del análisis, se considera que las notas periodísticas deportivas en español hacen uso de un abanico de conectores de todo tipo, a saber: aditivos, consecutivos, contraargumentativos, causales, condicionales y finales. Sin embargo, de acuerdo con los resultados que se desprenden del análisis del corpus, se advierte que hay un considerable número de conectores que no son empleados por los periodistas a cargo de las notas deportivas, pues hay una tendencia a utilizar ciertos conectores que resultan más comunes y, posiblemente, de más fácil comprensión para el lector de las notas.

En el apartado del análisis se pudo apreciar que los conectores finales fueron los más frecuentes con 520 casos (tokens), lo que representa el 35.57\% del corpus, pero de los cuales sólo se registraron cuatro tipos (types): para (que), por, con el fin de y con miras a. De hecho, dichos conectores junto con los de condición resultaron los menos variados del corpus. Los segundos conectores más frecuentes fueron los contraargumentativos con 499 casos, esto es $34.13 \%$, y 15 tipos: pero, mientras que, sin embargo, a pesar de, pese a, sino, no obstante, en lugar/vez de, con todo, aun así, si bien, en tanto que, después de todo, por el contrario y sea como sea. De hecho, los conectores contraargumentativos resultaron por mucho los más variados del corpus.

Luego de los conectores finales y contraargumentativos se ubican los conectores de causa con 238 casos, es decir 16.28\%, y nueve tipos: porque, ya que, pues, 
gracias a, por eso, a causa de, puesto que, dado que y por el hecho que. Los aditivos resultaron los siguientes en frecuencia con 91 casos, esto es 6.22\%, y seis tipos: además, incluso, asimismo, igualmente, de igual maneralmodolforma y es más. Cabe señalar que si bien la adición es una relación lógica muy común en casi cualquier género textual incluyendo las notas periodísticas deportivas, en el corpus utilizado no resultaron las más frecuentes; se considera que esto se debe a que no se incluyó la conjunción $y$ que habría elevado significativamente las frecuencias.

A continuación aparecen los conectores consecutivos que no resultaron tan recurrentes en el corpus, pues se registraron apenas 63 casos, lo que representa el 4.31\% del corpus, y seis tipos: por lo que, por elloleso, asi que, entonces, de maneral modo que y de ahi que. Finalmente, los conectores de condición resultaron los menos frecuentes en el corpus, ya que se registraron únicamente 51 casos, es decir apenas 3.49\%, y sólo cuatro tipos: si, cuando, siempre y cuando y siempre que. La Tabla VII sintetiza mejor los resultados antes presentados.

Tabla VII. Síntesis de las frecuencias de los conectores.

\begin{tabular}{|l|c|c|}
\hline Conectores & $\begin{array}{l}\text { Casos } \\
\text { (tokens) }\end{array}$ & $\begin{array}{l}\text { Tipos } \\
\text { (types) }\end{array}$ \\
\hline Finales & $520(35.57 \%)$ & 4 \\
\hline Contraargumentativos & $499(34.13 \%)$ & 15 \\
\hline Causales & $238(16.28 \%)$ & 9 \\
\hline Aditivos & $91(6.22 \%)$ & 6 \\
\hline Consecutivos & $63(4.31 \%)$ & 6 \\
\hline Condicionales & $51(3.49 \%)$ & 4 \\
\hline Total & $1.462(100 \%)$ & 44 \\
\hline
\end{tabular}

En cuanto a los conectores específicos, el más recurrente en el corpus resultó el conector de finalidad para (que) con 512 frecuencias, lo que representa el 35.57\% del corpus completo. Cabe mencionar que de las 512 ocasiones que se encontró dicho conector, en sólo 39 llevaba la conjunción. En segundo lugar se ubica el conector contraargumentativo pero con 299 recurrencias, esto es $20.45 \%$ del corpus. En tercer lugar se encuentra el conector de causa porque con 88 frecuencias, lo que significa el 6.02\%. Enseguida se sitúa el conector aditivo además con 69 repeticiones, es decir $4.72 \%$. Posteriormente, se ubica otro conector de contraargumentación, éste fue mientras que con 52 recurrencias, lo que representa el 3.56\% del corpus. Otros conectores recurrentes resultaron ya que con 48 frecuencias, pues con 46, si con 42, sin embargo con 41 y a pesar de con 39. 
Con base en la Tabla VII y lo comentado en el párrafo anterior, se puede concluir que los periodistas deportivos muestran ciertas tendencias al momento de expresar la junción de cláusulas, pues las relaciones de finalidad y de contraargumentación parecen ser las predilectas al momento de redactar sus notas. Por un lado, se observó que la finalidad suele expresarse mediante un conector privilegiado, éste es para que va seguido de un sintagma de infinitivo y con menor asiduidad la locución para que + cláusula en presente de subjuntivo. Es interesante observar cómo este conector resulta por mucho el más utilizado por los periodistas, pues se le prefiere por encima de todo un abanico de conectores finales tales como a fin de, con el propósito de, con el objeto de, con vistas a, con idea de, con intención de, a efectos de, con motivo de y con pretexto de que no se emplean en ningún texto del corpus. Por otro, en la contraargumentación se ubicó el conector pero como el preferido de los periodistas deportivos; dicho conector es considerado parentético (Montolío, 2001) y de oposición (Calsamiglia y Tusón, 2002). Cabe señalar que en este rubro los periodistas también recurren regularmente a otros conectores como mientras que, sin embargo, a pesar de, pese a, etc.

Así como se detectaron tendencias hacia la expresión de ciertas relaciones lógicas, también se ubicaron aquellas relaciones que los periodistas deportivos despliegan con menor recurrencia. Dichas relaciones son de consecuencia con 4.31\% y de condición con apenas $3.49 \%$ del corpus. Por un lado, se considera que las primeras relaciones no resultan tan frecuentes, porque en el marco de situaciones de causa-consecuencia, los periodistas prefieren hacer hincapié en la relación de causa a través de conectores como porque, ya que y pues en lugar de hacer uso de conectores que evidencian la consecuencia. Por otro, se concluye que los conectores condicionales resultan los menos recurrentes del corpus porque su propósito consiste en dar cuenta de situaciones posibles e inexistentes (Montolío, 1999), de tal suerte que no cumplen con la función principal de la nota periodística en sí, cuya intención es dar cuenta de situaciones que acontecen en la realidad.

Por último, es importante comentar que si bien se ha logrado el propósito de identificar los conectores discursivos más frecuentemente empleados en un corpus constituido por 300 notas periodísticas deportivas de diferentes diarios de habla hispana, se tiene conciencia de que dichos hallazgos no son categóricos y distan mucho de serlo. Por tal motivo, se considera conveniente que se siga investigando respecto a los conectores discursivos en diversos géneros textuales a fin de evidenciar las relaciones lógicas a las que los usuarios del español recurren con mayor asiduidad. Asimismo, es sustancial seguir estudiando los rasgos que caracterizan los diferentes discursos. En el presente estudio el foco fue el discurso periodístico deportivo, pero en definitiva, debe señalarse que existe una diversidad de discursos que sin duda alguna sería muy interesante estudiar desde una perspectiva de las relaciones lógicas y la junción de cláusulas. 


\section{REFERENCIAS}

Algeo, James E. (1973). The concessive conjunction in Medieval Spanish and Portuguese; its function and development. Romance Philology, 26 (3), 532-543.

Álvarez, Alfredo I. (1999). Las construcciones consecutivas. En Bosque, I. y Demonte, V. (Eds.) Gramática Descriptiva de la Lengua Española. Madrid, España: Espasa-Calpe: 3739-3804.

Anthony, Laurence. (2004). AntConc: A Learner and Classroom Friendly, MultiPlatform Corpus Analysis Toolkit. IWLeL: An Interactive Workshop on Language e-Learning, 7-13.

Calsamiglia Blancafort, Helena y Tusón Valls, Amparo. (2002). Las cosas del decir. Manual de análisis del discurso. Barcelona, España: Ariel.

De Beaugrande, Robert y Dressler, Wolfgang Ulrich. (1997). Introducción a la lingüistica del texto. Barcelona, España: Ariel.

De Vega, Manuel (2005). El procesamiento de oraciones con conectores adversativos y causales. Cognitiva, 17, 85-108.

Domínguez García, Noemí. (1997). Conectores: estado de la cuestión. Interlingüistica, 6, 33-38.

Flamenco García, Luis. (1999). Las construcciones concesivas y adversativas. En Bosque, I. y Demonte, V. (Eds.) Gramática Descriptiva de la Lengua Española. Madrid, España: Espasa-Calpe: 3805-3878.

Franco M., Antonio. (2004). El discurso periodístico a través de la lingüística textual. Opción, 43, 77-100.

Galán Rodríguez, Carmen. (1999). La subordinación causal y final. En Bosque, I. y Demonte, V. (Eds.) Gramática Descriptiva de la Lengua Española. Madrid, España: Espasa-Calpe: 3597-3642.

Halliday, Michael Alexander Kirkwood y Hasan, Ruqaiya. (1976). Cohesion in English. London, England: Longman.

Lozano, Jorge; Peña-Marín, Cristina y Abril, Gonzalo. (1999). Análisis del discurso. Hacia una semiótica de la interacción textual. Madrid, España: Cátedra.

Martín Zorraquino, María Antonia y Portolés Lázaro, José. (1999). Los marcadores del discurso. En: Bosque, I. y Demonte, V. (Eds.) Gramática descriptiva de la lengua española. Madrid, España: Espasa-Calpe: 4051-4213.

Medina Cano, Federico. (2010): Los narradores deportivos y sus epopeyas cotidianas. En S. Martínez (coord.). Fútbol-espectáculo, Cultura y Sociedad. Ciudad de México, México: Afínita Editorial/ Universidad Iberoamericana: 157-207.

Montolío, Estrella. (1999). Las construcciones condicionales. En: Bosque, I. y Demonte, V. (Eds.) Gramática descriptiva de la lengua española. Madrid, España: Espasa-Calpe: 3643-3737.

Montolío, Estrella. (2001). Conectores de la lengua escrita. Barcelona: Ariel. 
Murray, John D. (1997). Connectives and narrative text: The role of continuity. Memory and Cognition, 25 (2), 227-236.

Quintero Ramírez, Sara. (2013). Técnicas de nexión y junción en textos periodísticos deportivos. Revista Electrónica de Lingüistica Aplicada RAEL, 12, 1-15.

Sánchez Avendaño, Carlos. (2005). Los conectores discursivos: su empleo en redacciones de estudiantes universitarios costarricenses. Filología y Lingüística, XXXI (2), 169-199.

Soria, Claudia. (2005). Constraints on the Use of Connectives in Discourse. Manuscrito no publicado. Istituto de Linguistica Computazionale (CNR), Pisa, Italia.

Ubidia Rodríguez, Mayra Sofía y Enrique Pereira, Francisco Javier. (2012). Análisis del discurso de la narración deportiva en las radios deportivas de Quito (La Red y Rumba Deportiva), Tesis de Licenciado en Comunicación Social. Quito, Ecuador: Universidad Politécnica Salesiana. 